\title{
CATASTROPHIC OVERTURN OF THE EARTH'S MANTLE DRIVEN BY MULTIPLE PHASE CHANGES AND INTERNAL HEAT GENERATION
}

\author{
Stuart A. Weinstein \\ Department of Geological Sciences, The University of Michigan
}

\begin{abstract}
The effects of phase changes and strong internal heat generation may combine to bring about brief, but extremely intense episodes of rapid thermal convection in the Earth's mantle. Numerical calculations using realistic thermodynamic properties for the exothermic Olivine $\rightarrow$ Spinel and endothermic Spinel $\rightarrow$ Perovskite + Magnesiowustite phase transitions suggest the transition region of the Earth's mantle may act as a capacitor for subducting slabs. Slab material accumulates in the transition region until a threshold level of thermal buoyancy is reached, and is then rapidly discharged into the lower mantle. In my calculations, this occurs as a catastrophic burst of convection lasting $\sim 10 \mathrm{Myr}$, with elevated heat transfer rates lasting $\sim 100$ Myr. Such episodes may be analogs to superplume activity which has been hypothesized to give rise to an intense episode of intra-plate volcanism and stabilize the geodynamo against reversals. The topography on the two phase change boundaries is found to be negatively correlated, with the correlation becoming more negative during periods of rapid convection. Furthermore, the results of this study suggest the transition region of the Earth's mantle could be $-250 \mathrm{~K}$ cooler on average, and consequently more viscous, than the surrounding mantle.
\end{abstract}

\section{Introduction}

The effects of multiple phase transitions on thermal convection in the mantle is an important dynamical problem that has been receiving much attention [Liu et al., 1991, Zhao et al., 1992, Honda et al., 1992, Peltier and Solheim, 1992, Steinbach and Yuen, 1992]. These studies show how multiple transitions filter ascending plumes, [Zhao et al., 1992], affect secondary instabilities arising from an internal thermal boundary layer [Liu et al., 1991], control the thermal spectrum of the transition zone [Peltier and Solheim, 1992, Zhao et al., 1992] and influence the large scale structure of convection [Peltier and Solheim, 1992, Steinbach and Yuen, 1992, Zhao et al., 1992]. This study focuses on the combined effects of phase changes and internal heat generation; together these effects produce short periods of vigorous convection which could have played a fundamental role in Earth history.

\section{Model}

Our model consists of a 2-D, isoviscous, Newtonian, incompressible fluid layer heated from below and internally, with two isochemical phase changes representing the exothermic Olivine $\rightarrow$ Spinel and endothermic Spinel $\rightarrow$ Perovskite $+(\mathrm{Mg}, \mathrm{Fe}) \mathrm{O}$ phase transitions. The dimensionless

Copyright 1993 by the American Geophysical Union.

Paper number 93GL00044

0094-8534/93/93GL-00044\$03.00 governing equations for heat and momentum conservation are

$$
\begin{aligned}
& \frac{\partial \mathrm{T}}{\partial \mathrm{t}}+\mathrm{J}(\Psi, \mathrm{T})=\nabla^{2} \mathrm{~T}+\mathrm{R} \\
& \nabla^{2} \omega_{y}=\operatorname{Ra}\left(1+\frac{\mathrm{Rp}_{1}}{\mathrm{Ra}} \gamma_{1} * \frac{\mathrm{d} \Gamma_{1}}{\mathrm{~d} \Pi}+\frac{\mathrm{Rp}_{2}}{\mathrm{Ra}} \gamma_{2} * \frac{\mathrm{d} \Gamma_{2}}{\mathrm{~d} \Pi}\right) \frac{\partial \mathrm{T}}{\partial \mathrm{x}} \\
& \nabla^{2} \Psi=\omega_{y} \\
& \mathrm{Ra}=\frac{\rho_{\mathrm{og}} \alpha \Delta \mathrm{TD}^{3}}{\kappa \mu} \quad \mathrm{R}=\frac{\varepsilon \mathrm{D}^{2}}{\mathrm{~K} \Delta \mathrm{T}} \quad \mathrm{Rp}_{1,2}=\frac{\mathrm{g} \Delta \rho_{12} \mathrm{D}^{3}}{\kappa \mu}
\end{aligned}
$$

where $T$ is the temperature, $\Psi$ is the streamfunction, $R$ is the dimensionless heat source, $\omega_{y}$ is the vorticity, $\Pi$ is the reduced pressure, $\mathbf{R a}$ is the Rayleigh number based on the imposed temperature difference $\Delta T, R_{p_{1,2}}$ are the phase change Rayleigh numbers, $\Gamma_{1}$ and $\Gamma_{2}$ are the phase functions determining the fraction of denser phase and $\gamma_{1}{ }^{*}$ and $\gamma_{2}{ }^{*}$ are the dimensionless Clapeyron slopes. The other symbols $\mathrm{D}, \mathrm{g}$, $\alpha, K, \mu, p_{o}, \Delta p_{1,2}, K$, and $\varepsilon$ are the layer thickness, acceleration of gravity, thermal expansion coefficient, thermal diffusivity, dynamic viscosity, density at the top of the layer, phase transition density changes, thermal conductivity and internal heat generation rate per unit volume. The subscripts 1,2 denote the exothermic and endothermic transitions, respectively. The phase functions are calculated using the method of Richter [1973] with a transition half-width constant of $43 \mathrm{~km}$.

All of the fluid boundaries are assumed to be stress-free and impermeable. In addition, the top and bottom boundaries are isothermal and the sidewalls are insulating. The boundary conditions are:

$$
\begin{array}{cc}
\Psi=\omega_{y}=\mathrm{T}=0 & {[\mathrm{z}=1]} \\
\Psi=\omega_{y}=0, \mathrm{~T}=1 & {[\mathrm{z}=0]} \\
\Psi=\omega_{y}=\frac{\partial \mathrm{T}}{\partial \mathrm{x}}=0 & {[\mathrm{x}=0,1]}
\end{array}
$$

Equations (1)-(3) are solved with respect to the boundary conditions (5)-(7) using the method of finite differences on a uniform mesh. There are $\mathbf{2 2 5}$ grid points in the vertical giving $12.8 \mathrm{~km}$ resolution when scaled to the thickness of the mantle. The equilibrium positions (when there is a conductive temperature profile) of the exothermic and endothermic phase transitions correspond to dimensional depths of $280 \mathrm{~km}$ and $460 \mathrm{~km}$ respectively. The initial temperature distribution consists of upper and lower boundary layers and has a mean dimensionless temperature of 0.5 . In order to avoid a long transient period, $R$ is set initially to 30 and then lowered to 20 after the average dimensionless temperature reached 0.6 .

Table 1 contains both the values used for the dimensionless parameters in equations (1)-(3) and the values of the constants that were used in calculating them. The values for the Clapeyron slopes and transition density changes used here are given by [Ito and Takahashi, 1989, Ito 
Table 1: Parameter Values

\begin{tabular}{|c|c|}
\hline Parameters & Values \\
\hline $\mathbf{R a}$ & $1.0 \times 10^{7}$ \\
\hline $\mathrm{Rp} 1$ & $1.67 \times 10^{7}$ \\
\hline Rp2 & $2.0 \times 10^{7}$ \\
\hline $\mathrm{R}$ & 20 \\
\hline$\gamma_{1}^{*}$ & .106 \\
\hline$\gamma_{2}^{*}$ & -.09 \\
\hline$\alpha$ & $2.0 \times 10^{-5} \mathrm{~K}^{-1}$ \\
\hline$\Delta \mathrm{T}$ & $2750 \mathrm{~K}$ \\
\hline$\rho_{0}$ & $3300 \mathrm{~kg} \mathrm{~m}^{-3}$ \\
\hline$\gamma_{1}$ & $3.6 \mathrm{MPa} \mathrm{K}^{-1}$ \\
\hline$\gamma_{2}$ & $-3.0 \mathrm{MPa} \mathrm{K}^{-1}$ \\
\hline$\Delta \rho_{1 /} \rho_{o}$ & .09 \\
\hline$\Delta \rho_{2} / \rho_{0}$ & .11 \\
\hline$\mu$ & $4.2 \times 10^{21} \mathrm{~Pa} \mathrm{~s}$ \\
\hline$\varepsilon$ & $1.9 \times 10^{-8} \mathrm{~W} \mathrm{~m}^{-3}$ \\
\hline$\kappa$ & $1.0 \times 10^{-6} \mathrm{~m}^{-3} \mathrm{~s}^{-1}$ \\
\hline $\mathrm{g}$ & $9.8 \mathrm{~m} \mathrm{~s}^{-2}$ \\
\hline D & $2.88 \times 10^{6} \mathrm{~m}$ \\
\hline
\end{tabular}

et al., 1990, Ringwood, 1991, Anderson, 1987]. The other constants are given values appropriate for the mantle.

\section{Results and Discussion}

The kinetic energy, volume, basal and surface averaged Nusselt number, and average transition depth time series obtained from a long integration of equations (1)-(3) are shown in Figures 1-3. These time series start approximately $\sim 2.5$ overturns after $R$ was lowered to 20 . The kinetic energy and Nusselt number time series are dominated by a few large peaks which occur during brief episodes of extreme heat and mass transfer when the fluid above the endothermic transition plummets through the transition destroying a predominantly layered convection pattern. The kinetic energy of the fluid layer varies by nearly four orders of magnitude and the volume averaged Nusselt number varies by more than two orders of magnitude. The peak values of the volume averaged Nusselt number exceed 500 with a maximum of 1230 and are an order of magnitude greater than their counterparts in the basal and surface averaged Nusselt number. During these brief episodes, a tremendous amount of potential energy is released in a short burst which then slowly dissipates through the boundary layers. This accounts for the discrepancy in size between the peak volume averaged and surface and basal averaged Nusselt numbers [Grossman and Lohse, 1991].

The episode which occurs at $t=0.009$ will be examined in detail and is shown in a sequence of temperature and streamfunction fields (Figure 4). During the first two time instants (Figures 4a, 4b) cold fluid accumulates from drifting instabilities off the top boundary-layer on the left side of the layer above the endothermic transition. Eventually enough buoyancy exists to overcome the stabilizing effect of the endothermic transition. When this occurs, a substantial fraction of the cold fluid above the endothermic transition catastrophically descends to the bottom of the layer at speeds exceeding $50 \mathrm{~cm} / \mathrm{yr}$ (Figure $4 \mathrm{c}$ ). The descent through the lower layer requires about $\sim 10 \mathrm{Myr}$. As the cold fluid descends the hot, lower part of the fluid layer moves upwards through the endothermic transition. This results in a rapid rise in the basal and surface averaged Nusselt numbers by a factor of $\sim 5$ (Figure $2 b$ ), and reduces the transition zone thickness (Figure 3). After the cold fluid has descended through the lower layer and spread across the base of the box (Figures $4 \mathrm{~d}-4 \mathrm{f}$ ), the pattern of convection again becomes predominantly layered. The horizontally averaged temperature profiles $(\langle T\rangle)$ now reflect the abundance of cold fluid on the bottom of the fluid layer and hot fluid above the endothermic transition (Figures $4 \mathrm{e}, 4 \mathrm{f}$ ). This thermal distribution excites the formation of instabilities in the lower and upper thermal boundary layers (Figure 4f) maintaining high heat transfer rates. The elevated heat transfer rates decay

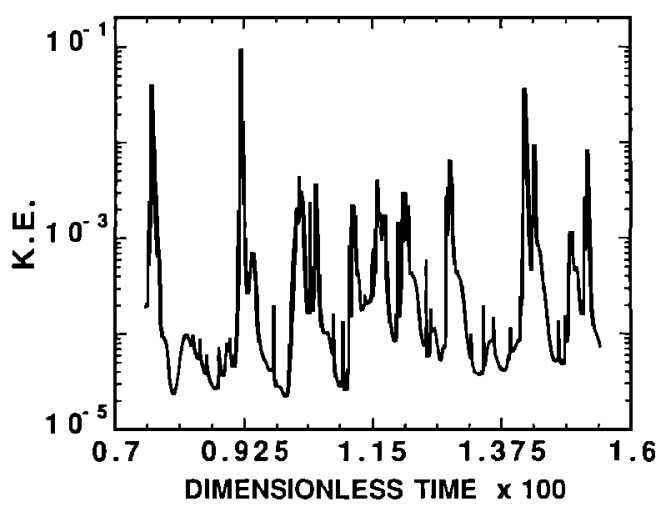

Fig. 1. Log of volume kinetic energy time series. The kinetic energy is normalized by $\mathrm{Ra}^{2}$. A dimensionless time interval of .001 corresponds to $-260 \mathrm{Myr}$.
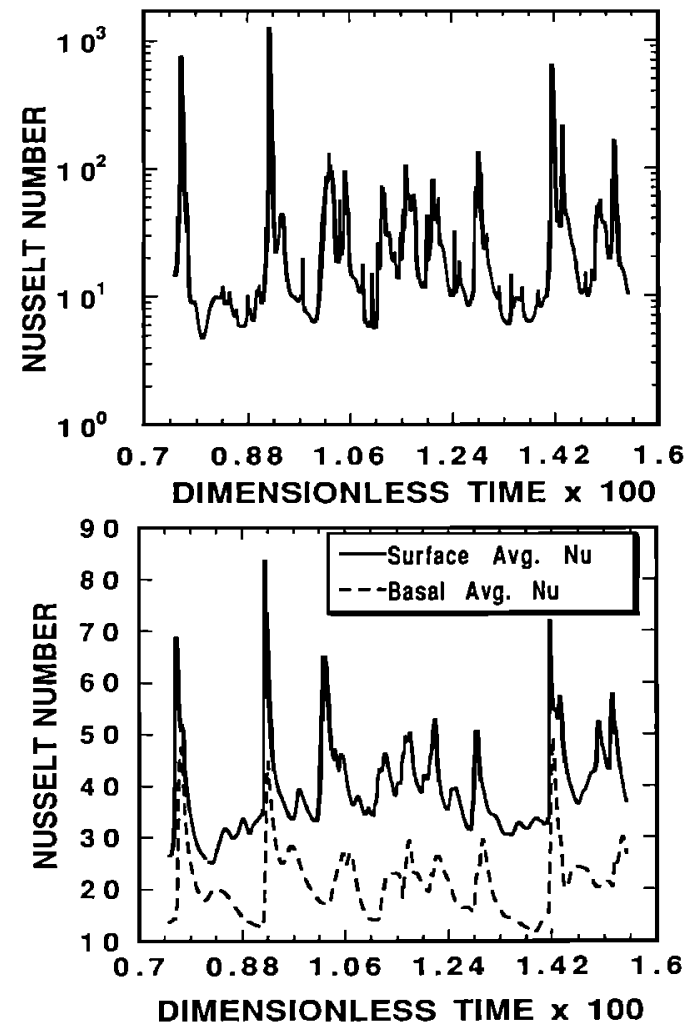

Fig. 2. (a) Log of volume averaged Nusselt number time series. (b) Linear plot of basal and surface averaged Nusselt number time series. 


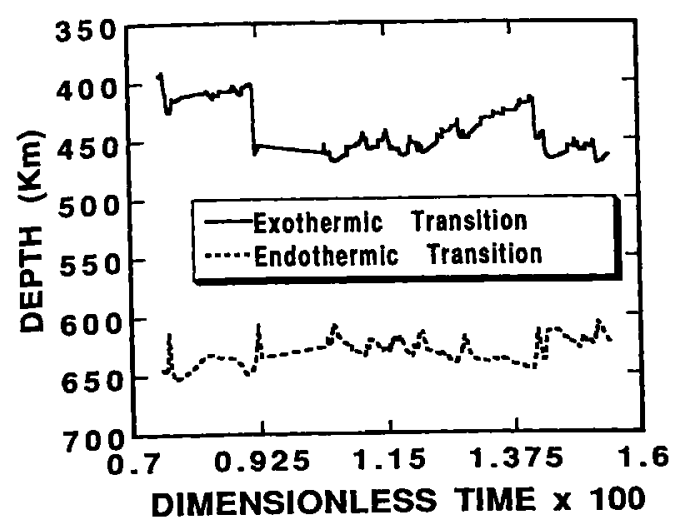

Fig. 3. Time series of average phase transition depths. The phase transition depth is the depth at which $\Gamma_{1,2}=.5$.

to background levels over $\sim 100$ Myr. Based on the surface and basal Nusselt number time series during the time interval displayed in Figures 1-3, radiogenic heating contributes $40 \%$ $60 \%$ of the surface heat flow.

Intermittent layered convection, in which a layered convection pattern is disrupted by occasional strong downwellings was first described by Machetel and Weber [1991]. The episodes found in this study are qualitatively similar to, but clearly more intense, than those found in the spherical axisymmetric calculations of Machetel and Weber [1991], and those found recently in the 3-D Cartesian studies

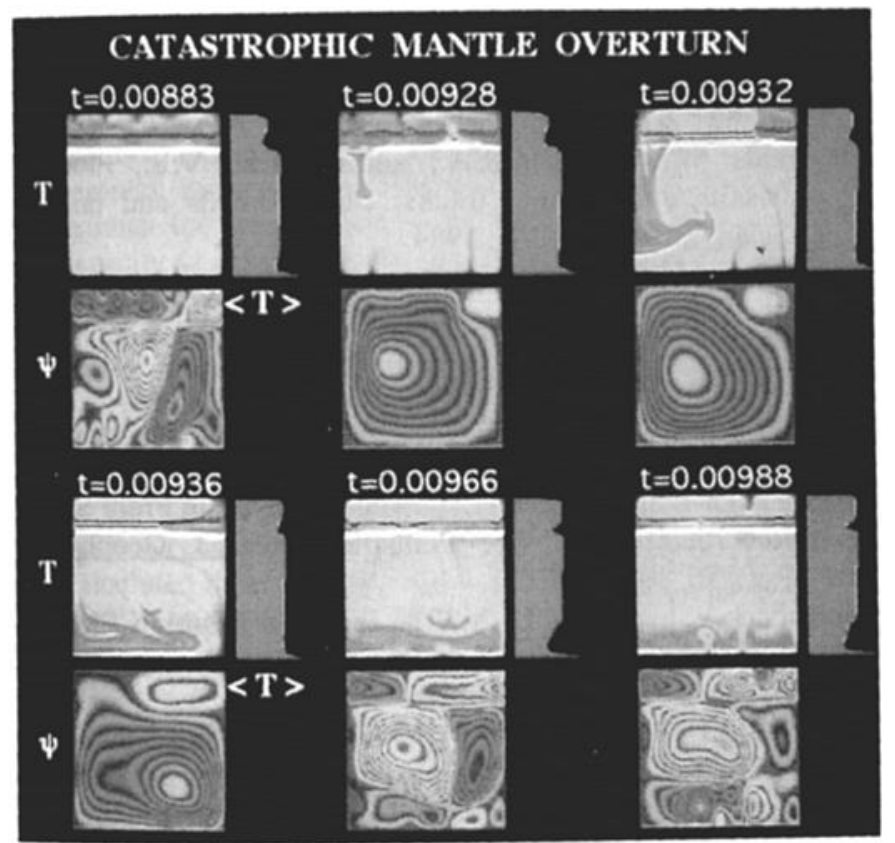

Fig. 4. Sequence of temperature and streamfunction fields spanning the time interval $0.00883-0.00988$. In the temperature fields, blue and red represent cold and hot fluid respectively. In the streamfields, blue and red indicate fluid moving counter-clockwise respectively. The histogram to the right of the temperature fields represents the horizontally averaged temperature distribution. The black and white lines are the .2 and .8 contours of the $\Gamma_{1}$ and $\Gamma_{2}$ functions respectively. by Honda et al., [1992, 1993] and the 3-D spherical calculations of Tackley et al., [1992]. While the effects of compressibility (e.g., viscous dissipation, latent heat) may effect these episodes, it is clearly not responsible for them. The greater intensity of the catastrophic events found in this study may be due to the effects of small aspect-ratio, the presence of the exothermic phase transition or due to the effects of greater internal heat generation.

For the time interval used in Figures 1-3, the transition zone width thickness varies between $138 \mathrm{~km}$ and $255 \mathrm{~km}$, with an average of $177 \mathrm{~km}$. The mean depths are $449.5 \mathrm{~km}$ and $626.5 \mathrm{~km}$ for the exothermic and endothermic transitions. Present estimates of depths of $410 \mathrm{~km}$ and $660 \mathrm{~km}$ for these transitions in the Earth are bracketed by values obtained in the course of the calculation. During the episodes of rapid convection, the transition zone thickness decreases rapidly, by nearly $100 \mathrm{~km}$, and slowly increases there after.

Revenaugh and Jordan [1991] found negative linear correlation coefficients between travel times through the transition zone and lower mantle ( $r=-0.65)$, between travel times through the transition zone and upper mantle $(r=-0.78)$ and a positive correlation between travel times through the upper mantle and travel times through the lower mantle $(r=0.35)$. These correlations are primarily due to topography on the $410 \mathrm{~km}$ and $670 \mathrm{~km}$ discontinuities and are consistent with the behavior expected for phase transitions with Clapeyron slopes of different sign. Figure 5 shows the variation of the linear correlation coefficient of the topography on the two phase transitions and kinetic energy over a time interval centered on a catastrophic episode occurring at 0.0141 dimensionless time. The correlation coefficients are always negative and range in value from -0.40 to -0.88 . When the kinetic energy is large, the correlation coefficient is more negative. This relationship exists during periods of rapid convection, because there is a large fluid flux through both phase transitions. At other times when there is a significant amount of layering, the correlation is much weaker. Perhaps a global determination of $r$ for the $410 \mathrm{~km}$ and $670 \mathrm{~km}$ discontinuities can be used as a barometer for plume activity in the mantle.

Recently, Larson and Olson [1991] hypothesized that superplume activity during the Cretaceous stabilized the

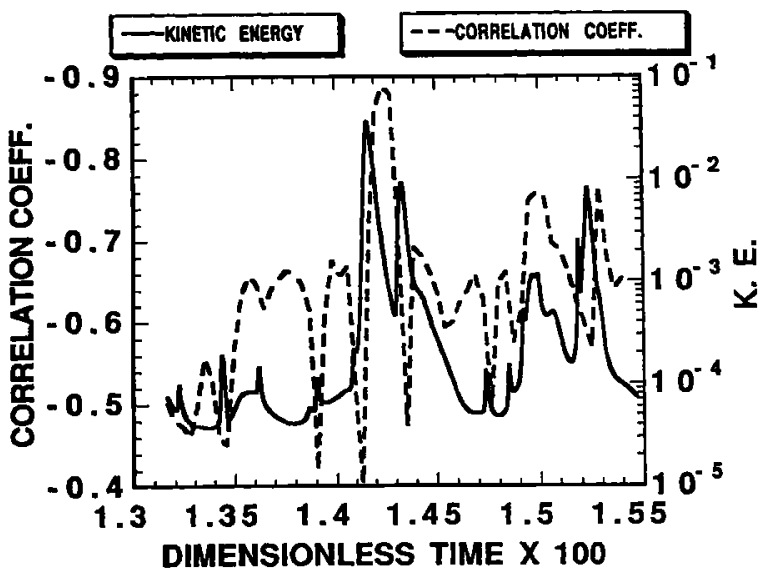

Fig. 5. Plot showing the variation of the linear correlation coefficient $(R)$ and kinetic energy in a time window centered on the event at .0141 dimensionless time. 


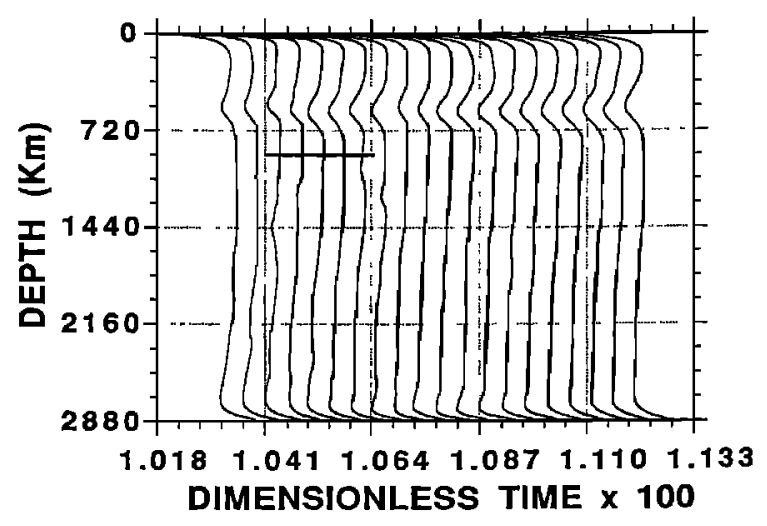

Fig. 6. Sequence of horizontally averaged temperature profiles spanning the time interval $.01018-.01330$. The horizontal bar indicates a variation in $\mathrm{T}$ of 1.0 .

geodynamo against reversals and was responsible for the Cretaceous superchron. Their model is comprised of the plume model of Richards et al., [1989] and dynamo theory. The basic premise is that a plume head detaching from a thermal boundary layer at the core-mantle boundary (CMB) will greatly increase the heat flow at the CMB and stabilize the geodynamo against reversals. After 10-20 Myr the plume head will reach the surface giving rise to flood basalts. A prediction of this model is that the superchron begins before flood basalt production commences. The catastrophic episodes observed in the calculation presented here may also give rise to superchrons. In this model, heat flow at the CMB increases due to the cold material which falls on it. Perhaps even more important is the difference in timing between the generation of flood basalts and the onset of the superchron. Figures $4 b-4 c$ clearly show the injection of hot fluid into the upper layer before the cold material reaches the bottom. Therefore, in this model flood basalt generation begins before the onset of the superchron. I suggest that perhaps a superchron indicates a period where portions of the upper mantle became gravitationally unstable and precipitated a transition from two-layer convection to vigorous whole mantle convection.

The general thermal structure can be understood from an examination of the horizontally averaged temperature profiles obtained during a period of low activity. The profiles shown in Figure 6 span a period of time lasting about 1 overturn. The profiles show a low temperature zone between the phase transitions. This feature is also present in the calculations of Zhao et al., [1992]. The cold zone develops as cold, descending flow is nearly prevented from penetrating the endothermic transition and spread out over the endothermic transition boundary. The temperature contrast between the cold zone and surrounding regions is $\sim 250 \mathrm{~K}$. The cold zone still exists, to a lesser degree, even during episodes of rapid convection (Figure 4 ).

Acknowledgment. I thank Mike Gurnis for his help and comments and the Pittsburg Supercomputer Center. Computational facilities at Michigan are partly funded by NSF EAR-8937164. S. Weinstein is supported by a 1991 NSF Earth Sciences Postdoctoral Research Fellowship, grant NSF EAR-9102808.

\section{References}

Anderson, D.L., Thermally induced phase changes, lateral heterogeneity of the mantle, continental roots and deep slab anomalies, I. Geophys. Res., 92, 13968-13980, 1987

Grossman S., and Lohse D., Fourier-Weierstrass Mode Analysis for Thermally Driven Turbulence, Phys. Rev Lett., 67, 445-448, 1991

Honda S., Balachandar S., Yuen D. A. and Reuteler D., Dynamics of Three-Dimensional mantle Convection with an Endothermic Phase Transition, Geophys. Res. Lett., in press, 1992

Honda S., Balachandar S., Yuen D. A. and Reuteler D., Three-Dimensional Onstabilities of Mantle Convection with Multiple Phase Transitions, Science, in press, 1993

Ito E. and Takahashi E., Postspinel transformation in the system $\mathrm{Mg}_{2} \mathrm{SiO}_{4}-\mathrm{Fe}_{2} \mathrm{SiO}_{4}$ and some geophysical implications, J. Geophys. Res. 94, 10637-10646, 1989

Ito E., Akaogi M., Topor L., and Navrotsky, A., Negative pressure-temperature slopes for reactions forming $\mathrm{MgSiO}_{3}$ perovskite from calorimetry, Science, 249, $1275-1278,1990$

Larson R. L. and Olson P., Mantle plumes control magnetic reversal frequency, Earth Planet. Sci. Lett. 107, 437 447, 1991

Liu M., Yuen D. A. and Honda S., Development of Diapiric Structures in the Upper mantle Due to Phase Transitions, Science, 252, 1836-1839, 1991

Machetel $\mathbf{P}$, and Weber $\mathbf{P}$., Intermittent layered convection in a model with an endothermic phase change at $670 \mathrm{~km}$, Nature, 350, 55-57, 1991

Peltier W. R. and Solheim L. P., Mantle Phase Transitions and Layered Chaotic Convection, Geophys. Res. Lett. $19,321-324,1992$

Richards M.A., Duncan R.A., and Courtillot V.E., Flood basalts and hotspot tracks: Plume heads and tails, Science, 246, 103-107, 1989

Richter F. M., Finite amplitude convection through a phase boundary, Geophys. J. R. Astron. Soc.e 35, 265-276, 1973

Ringwood A. E., Phase transformations and their bearing on the constitution and dynamics of the mantle, Geochim. Cosmochim. Acta, 55, 2083-2110, 1991

Revenaugh J. and Jordan T. H., Mantle Layering From ScS Reverberations 2. The Transition Zone, J. Geophys. Res. 96, 19763-19780

Steinbach V. and Yuen D. A., The Effects of Multiple Phase Transitions on Venusian Mantle Convection, In Press Geophys. Res. Lett., 1992

Tackley P.J., Stevenson D.J., Glatzmeir G.A., and Schubert G., Effects of an endothermic phase transition at $670 \mathrm{~km}$ depth on spherical mantle convection, Nature, in press, 1992

Zhao W., Yuen D. A. and Honda S., Multiple phase transitions and the style of mantle convection, Phys. Earth and Planet. Int. 72, 185-210, 1992

S. A. Weinstein, Department of Geological Sciences, The University of Michigan, Ann Arbor, MI 48109-1063.

Received: November 24, 1992

Accepted: December 15, 1992 